\title{
Effects of Vitamin D Supplementation on Omentin-1 and Spexin Levels, Inflammatory Parameters, Lipid Profile, and Anthropometric Indices in Obese and Overweight Adults with Vitamin D Deficiency under Low-Calorie Diet: A Randomized Placebo Controlled Trial
}

\author{
Elhameh Cheshmazar $\mathbb{D}^{1},{ }^{1}$ Agha Fatemeh Hosseini $\mathbb{D}^{2},{ }^{2}$ Bahareh Yazdani $\mathbb{D}{ }^{3}{ }^{3}$ \\ Elham Razmpoosh $\mathbb{D}^{4,5}$ and Mitra Zarrati $\mathbb{D D}^{1}$ \\ ${ }^{1}$ Department of Nutrition, School of Public Health, Iran University of Medical Sciences, Tehran, Iran \\ ${ }^{2}$ Department of Biostatistics, School of Public Health, Iran University of Medical Sciences, Tehran, Iran \\ ${ }^{3}$ Neonatal Screening Department of Nilou Laboratory, Tehran, Iran \\ ${ }^{4}$ Nutrition and Endocrine Research Center, Research Institute for Endocrine Sciences, \\ Shahid Beheshti University of Medical Sciences, Tehran, Iran \\ ${ }^{5}$ Integrative Oncology and Quality of Life Department, Breast Cancer Research Center, Motamed Cancer Institute, ACECR, \\ Tehran, Iran \\ Correspondence should be addressed to Mitra Zarrati; zarrati_ms@yahoo.com
}

Received 2 April 2020; Revised 13 October 2020; Accepted 28 October 2020; Published 11 November 2020

Academic Editor: Tadaaki Satou

Copyright ( 2020 Elhameh Cheshmazar et al. This is an open access article distributed under the Creative Commons Attribution License, which permits unrestricted use, distribution, and reproduction in any medium, provided the original work is properly cited.

\begin{abstract}
Background and Aims. Improved vitamin D levels can have a favorable effect on some metabolic variables. The objective of the current study was to determine the effects of vitamin D supplementation during a weight-loss intervention on the levels of omentin-1, spexin, lipid profiles, and inflammatory factors in obese and overweight participants. Methods and Materials. In this double-blind placebo-controlled randomized clinical trial, 70 overweight and obese participants with vitamin $\mathrm{D}$ deficiency $(25(\mathrm{OH}) \mathrm{D} \leq 20 \mathrm{nmol} / \mathrm{L})$ were assigned into the intervention (a daily dose of 2,000 IU vitamin $\mathrm{D}+$ low-calorie diet) and placebo (placebo + low-calorie diet) groups for 8 weeks. Anthropometric parameters, serum levels of 25-hydroxy vitamin D (25(OH)D), lipid profiles, omentin-1 and spexin levels, high-sensitivity C-reactive protein (hs-CRP), and soluble intercellular adhesion molecule-1 (sICAM-1) concentrations were assessed before and after the intervention. Results. Vitamin D supplementation after the intervention led to a significant decrease in triglycerides (TG) $(P=0.02)$, very-low-density lipoprotein-cholesterol (VLDL-C) $(P=0.02)$, and hs-CRP $(P=0.03)$ concentrations and a significant increase in the serum vitamin $\mathrm{D}$ level $(P<0.001)$. Furthermore, after adjusting for baseline values, age, and baseline BMI, the levels of serum high-density lipoprotein-cholesterol $($ HDL-C) $(P=0.01)$ increased significantly, and a significant reduction was observed in the concentration of sICAM-1 $(P=0.01)$ in the intervention group. However, we did not find any significant difference in serum omentin-1 and spexin concentrations between the groups after intervention. Conclusions. Vitamin D supplementation along with a low-calorie diet (LCD) program for 8 weeks significantly decreased the inflammatory markers in obese individuals, while it did not alter serum omentin-1 and spexin concentrations.
\end{abstract}




\section{Introduction}

Obesity is now a considerable public health problem, which is mainly caused by genetic factors, increased calorie intake, and the lack of physical activity [1]. Obesity and overweight have been proved to be associated with several comorbidities including diabetes mellitus, cardiovascular diseases (CVD), and different types of cancers such as breast and colon cancer, which are all caused by the inflammatory responses in the body [2]. Obesity and overeating are associated with accumulation and expansion of adipose tissue, so hypertrophic adipocytes may play an important role in the onset of inflammation by disrupting the balance of inflammatory and anti-inflammatory factors [3]. In fact, recent evidence has shown that vitamin $\mathrm{D}$ can modulate the secretions of many adipokines [4], which are mainly secreted by adipose tissue (AT). These adipokines mainly include spexin and omentin-1 which play an important role in several biological processes, such as regulating the uptake of longchain free fatty acids and the body fat storage, modulating food intake and energy metabolism [5], as well as regulating the anti-inflammatory, antidiabetic, antiatherogenic, and insulin-sensitizing effects in the body [6]. Therefore, there are many disorders known to be related to increased AT mass and the subsequent incidence of inflammation [7]. Different effects of vitamin D supplementation may be observed on adipokine homeostasis [8]. The presence of hypovitaminosis may impair the regulation of antioxidant and anti-inflammatory pathways [9]. Besides, it has been observed that a low-calorie diet could alleviate the inflammatory status and modulate the adipokine levels. Regulation of inflammatory and anti-inflammatory markers balance and increase insulin sensitivity after weight loss has been associated with a decrease in the prevalence of chronic diseases [10]. Improvements in adipokine profile with weight loss of $5-10 \%$ have been observed in previous studies [11-13]. There are previous animal and human studies on obesity and metabolic syndrome (MetS) that have reported various effects of vitamin D supplementation on some adipokines and the biomarkers of inflammation, blood pressure, glycemic control, and lipid profiles in obesity $[9,14]$.

To our knowledge, this is the first randomized doubleblind placebo-controlled trial of vitamin D supplementation on omentin-1 and spexin levels in obese and overweight adults during low-calorie diet with vitamin $\mathrm{D}$ deficiency.

Given the modulatory and anti-inflammatory effects of vitamin $\mathrm{D}$, we hypothesized that the low-calorie diet programs along with vitamin $\mathrm{D}$ supplementation could improve serum omentin-1 and spexin levels and modulate the inflammatory markers, lipid profiles, and anthropometric parameters in overweight and obese individuals. The purpose of this randomized placebo-controlled trial was to investigate the effects of a 2,000 IU of vitamin $\mathrm{D}_{3}$ supplementation per day for 8 weeks on some serum adipokines including omentin-1 and spexin levels, inflammatory markers, lipid profile, and anthropometric parameters along with a low-calorie diet program in overweight and obese participants who had vitamin D deficiency.

\section{Materials and Methods}

2.1. Study Design and Participants. This randomized doubleblind placebo-controlled clinical trial was conducted at the Nutrition Clinic of Iran University of Medical Sciences (IUMS). Seventy obese and overweight participants (25 men and 45 women) aged 20-50 years and with a baseline body mass index (BMI) of $25-35 \mathrm{~kg} / \mathrm{m}^{2}$ were recruited for the study. Inclusion criteria were having a serum $25(\mathrm{OH}) \mathrm{D}$ level of less than $20 \mathrm{ng} / \mathrm{mL}$, not taking any glucose and lipidlowering drugs, and willing to participate in the investigation. The exclusion criteria were having any metabolic diseases, diabetes or CVD, autoimmune diseases, any types of cancers, renal or hepatic and infection diseases, women with polycystic ovary syndrome, smoking, the alcohol consumption, taking immune system suppressor drugs in 6 months prior to the study initiation as well as having a weight loss diet program in 3 months prior to the research, taking any multimineral supplements or vitamin D supplements, the use of tanning bed during the study or in 3 months before the study initiation, and having no interests for participating in the research. The overall diagram of the study research is shown in Figure 1. At the onset of the study, all the participants were randomized into two groups of vitamin $\mathrm{D}$ intervention and placebo. Individuals were matched one by one according to their gender and BMI ranges. Randomization assignment was conducted using computer-generated random numbers as blindness by trained personnel. A balanced-blocked randomization list stratified by gender and BMI (block 1: BMI $25-29.9 \mathrm{~kg} / \mathrm{m}^{2}$ female, block 2: BMI: $30-35 \mathrm{~kg} / \mathrm{m}^{2}$ female, block 3: BMI $25-29.9 \mathrm{~kg} / \mathrm{m}^{2}$ male, and block $4:$ BMI $30-35 \mathrm{~kg} / \mathrm{m}^{2}$ male). Participants in the vitamin D group received an oral 2,000 IU of vitamin D supplement (Schiff \& Company, USA) per day for 8 weeks and the placebo group received capsules of edible paraffin oil for 8 weeks which were similar in their shapes and sizes to vitamin D supplements. To prevent the effects of seasonal variations on vitamin $\mathrm{D}$ levels, this study was conducted in the autumn and winter.

Both groups received a weight loss program at the baseline of the study. The related diet consisted of $12-15 \%$ calories of protein, $30-35 \%$ of fat, and $55-60 \%$ of carbohydrates which was estimated individually based on each participant's current age, weight, height, and gender. We asked every participant to come to the clinic every two weeks for weight monitoring and receiving their supplements for the upcoming two weeks. To increase compliance, all the participants received short messages on their cell phones every week to remind them of the adherence to their weight loss diet program and taking the supplements. To assess their dietary intakes, every participant completed a 3-day dietary record: at baseline, week 3 , and at the end of the trial. Modified Nutritionist-4 software program (First Databank, San Bruno, CA) was used to determine the macro- and micronutrient intakes. Physical activity was described as metabolic equivalents in hours per day and assessed three 


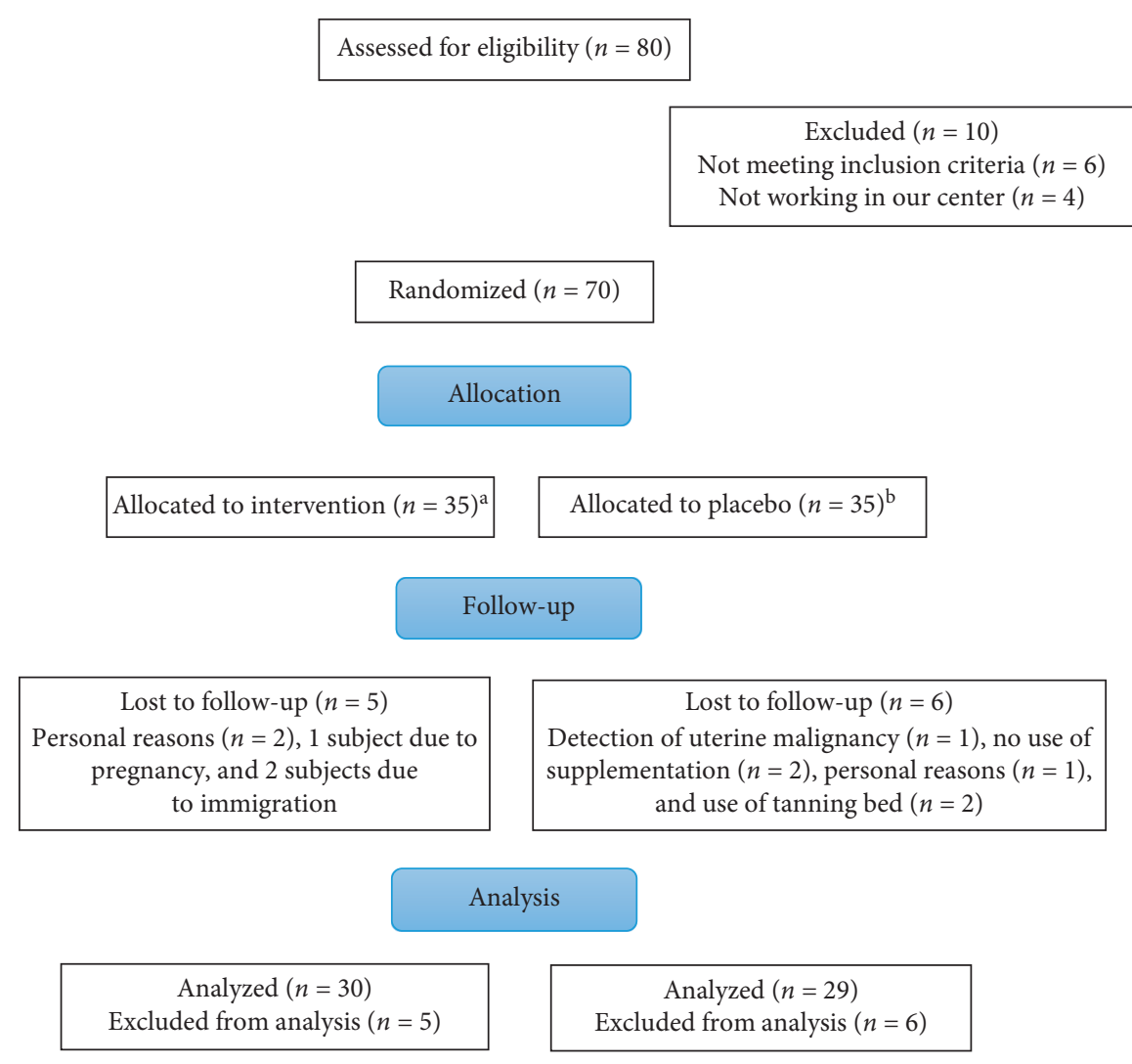

FIGURE 1: Summary of the patient flow diagram.

times by Long International Physical Activity (IPAQ) questionnaire during the study. Individuals were requested to have a 30-minute walk per day and not to consume any supplements other than the one provided to them by the investigators.

2.2. Sample Size Calculation. The mean \pm SD of omentin-1 and sICAM-1 were estimated from previous studies $[15,16]$. By assuming a type one error (a) of 0.05 and type two error (b) of 0.20 (power $=80 \%$ ), after considering a $10 \%$ dropout rate, a sample size of 35 participants per group was finally calculated.

2.3. Measurements. The primary outcomes were followed as adipokines including omentin-1 and spexin and inflammatory factors including hs-CRP and sICAM-1. Secondary outcomes were lipid profile consisting of LDL-C, HDL-C, TG, and TC, as well as VLDLD-C and anthropometric indices including BMI, weight, fat, and muscle percent, waist circumference (WC), and visceral fat (VF).

2.3.1. Assessment of Anthropometric Measures. Weight (nearest to $0.1 \mathrm{~kg}$ ) and height (nearest to $0.1 \mathrm{~kg}$ ) with light clothes were estimated. Fat percent and muscle percent were measured using a standard scale (Omron-Body Composition Monitor-BF511, Kyoto, Japan) and Seca Messband 206 in a fasting status at baseline and after the 8 weeks. Furthermore, BMI was calculated as weight in $\mathrm{kg}$ divided by the square of height in meter. WC and VF were estimated by ViScan device (Abdominal Fat Analyser AB-140, Tanita). The blood pressure of participants was measured by a digital blood pressure device (Omron, HEM-FL31) and we were confident that every individual was relaxed at least for 10-15 minutes before the measurement.

2.3.2. Sun Exposure Questionnaire. The participants' exposure to sunlight was assessed before and after the intervention through a validated questionnaire. In the questionnaire about the duration of sunlight exposure (the average minutes (min)/hours (h) of a usual day in the previous week), use of sunscreen, the types of coating, and the noncovered part of the body during sun exposure were asked [17]. The duration of sun exposure was classified as 0-10 $\mathrm{min} ; 10 \mathrm{~min}-1 \mathrm{~h} ; 1-2 \mathrm{~h}$; and $>2 \mathrm{~h}$. Moreover, the time of exposure to sunlight during a day was also asked and classified as follows: 7 am-10 am; 10 am-3 pm; and 3 pm-5 pm. Because of the intense sunlight, the highest score was the exposure of sunlight at 10-3 o'clock [18].

2.3.3. Biochemical Assessment. Blood samples (10 mL) were taken from every participant after overnight fasting at baseline and at the end of 8 weeks at the nutrition clinic of IUMS. 25(OH)D concentration was evaluated using a commercial ELISA kit (Immunodiagnostic Systems). The inter- and intra-assay coefficients of variability $(\% \mathrm{CV})$ for serum $25(\mathrm{OH}) \mathrm{D}$ tests ranged from $5 \%$ to $7.5 \%$. Serum spexin 
and omentin-1 (MyBioSource, San Diego, USA), sICAM-1 (Eagle Biosciences, USA) levels were quantified using enzyme-linked immunosorbent assay (ELISA) kits with interand intra-assay $\% \mathrm{CV}$ of 8.5 and $9.6 \%$, respectively. To determine the levels of fasting blood sugar (FBS), serum TG, TC, LDL-C and HDL-C, VLDL-C, and hs-CRP, we used related enzymatic kits (DIALAB Kit, Fara Samed Company, Iran).

2.4. Statistical Analysis. Data were statistically analyzed using the Statistical Package for Social Sciences (SPSS, version 22, Chicago, IL, USA). Using the one-sample Kolmogorov-Smirnov test, it was found that all primary and secondary data had normal distribution. Independent sample $t$-test was used for the comparison of continuous variables, dietary intakes, and anthropometric measures and Pearson's chi-square test for comparing the categorical variables between the 2 groups. We applied paired-sample $t$ test to establish within-group differences (pre- and posttreatment). Independent $t$-test was used to evaluate the effect of vitamin D supplementation on adipokines, inflammatory markers, and lipid concentrations in the intervention and placebo groups. The results were considered significant if the $P$ value was $<0.05$. To assess the effects of some confounders, we adjusted all analyses using ANCOVA test.

2.5. Ethical Approval. The study protocol was approved by the ethics committee of IUMS and registered at the Iranian Registry of Clinical Trial. This investigation was conducted according to the principles of the Declaration of Helsinki and carefully explained to all participants before obtaining the informed consent form.

\section{Results}

Of the 80 individuals screened for eligibility, 70 participants met the inclusion criteria and were allocated equally into 2 groups of 35 . However, $84.2 \%$ of all the participants including 29 individuals in the placebo and 30 individuals in the vitamin D group completed the study (Figure 1). In fact, 5 participants in the vitamin $\mathrm{D}$ group were lost to follow-up due to personal reasons $(n=2)$, pregnancy $(n=1)$, and immigration $(n=2)$. In addition, the other 6 participants who were lost to follow-up in the study were in the placebo group, and it was due to uterine malignancy $(n=1)$, personal reasons $(n=1)$, use of tanning bed $(n=2)$, and the lack of sufficient vitamin D supplement consumption $(n=2)$. No adverse effects of interventions were reported. General characteristics are shown in Table 1. The mean age and height of all patients were estimated to be $37.57 \pm 8.5$ years and $159.8 \pm 31.11 \mathrm{~cm}$, respectively.

3.1. Body Composition. In both groups, anthropometric indices (height, body weight, BMI, fat percent, and WC) except muscle percent and VF significantly decreased after 8 weeks of intervention, but changes between vitamin $\mathrm{D}$ and placebo groups were not significant. Systolic blood pressure
TABLE 1: General characteristics of study participants.

\begin{tabular}{|c|c|c|c|}
\hline & $\begin{array}{l}\text { Placebo group }^{1} \\
\quad(n=29)\end{array}$ & $\begin{array}{c}\text { Vitamin D group }^{2} \\
\quad(n=30)\end{array}$ & $P^{*}$ \\
\hline $\operatorname{Age}(y)^{\mathrm{a}, \mathrm{e}}$ & $36.82 \pm 7.65$ & $38.30 \pm 9.34$ & 0.5 \\
\hline Gender $^{\mathrm{d}}$ & & & 0.3 \\
\hline Female $^{\mathrm{b}}$ & $21(72.4)$ & $18(60)$ & \\
\hline Male ${ }^{\mathrm{b}}$ & $8(27.6)$ & $12(40)$ & \\
\hline Height $(m)^{\mathrm{a}, \mathrm{e}}$ & $154.5 \pm 143.13$ & $165.03 \pm 9.17$ & 0.1 \\
\hline \multicolumn{4}{|c|}{ Sun exposure (score) ${ }^{\mathrm{f}}$} \\
\hline Baseline $^{c}$ & $4.4(1.8,6.9)$ & $4.9(2.1,7)$ & 0.7 \\
\hline $\begin{array}{l}\text { End of the } \\
\text { trial }^{c}\end{array}$ & $3.9(1,7)$ & $4.4(1.7,5.8)$ & 0.8 \\
\hline \multicolumn{4}{|c|}{ Physical activity (IPAQ-METS score) ${ }^{g}$} \\
\hline Baseline $^{c}$ & $630(443,1299)$ & $682.5(470.25,1903.5)$ & 0.8 \\
\hline $\begin{array}{l}\text { End of the } \\
\text { trial }^{\mathrm{c}}\end{array}$ & $600(393.5,1400)$ & $827.5(415.7,1925)$ & 0.5 \\
\hline
\end{tabular}

${ }^{1}$ Received placebo per day during 8 weeks. ${ }^{2}$ Received 2,000 IU vitamin D per day during 8 weeks. ${ }^{*}$ Significant difference $(P<0.05)$. ${ }^{a}$ Data are presented as means $\pm \mathrm{SDs}$ and frequency $(\%)^{\mathrm{b}}$ or median (interquartile range) ${ }^{\mathrm{c}}$. ${ }^{\mathrm{d}}$ Obtained from chi-square. ${ }^{\mathrm{e}}$ Obtained from independent sample Student's $t$-test. ${ }^{\text {f } O b t a i n e d ~ f r o m ~ F i s h e r ' s ~ e x a c t ~ t e s t . ~}{ }^{g}$ Obtained from Mann-Whitney $U$ test.

(SBP) and diastolic blood pressure (DBP) were not significant within and between the two groups before and after the treatment. Table 2 summarizes the selected data of participants at the beginning and at the end of the study.

3.2. Food Intake. According to the 3-day dietary records, no statistically significant differences were observed between the two groups in terms of macro- and micronutrients (Table 3).

At the end of the study, it was found that dietary intakes of vitamin D (independent of vitamin D supplementation) were slightly diminished in both groups without any significant differences between the groups (Table 3).

3.3. Main Variable. After the 8 weeks of vitamin D supplementation, serum $25(\mathrm{OH}) \mathrm{D}$ concentrations increased in the vitamin $\mathrm{D}$ group (vitamin $\mathrm{D}$ vs. placebo groups: $16.8 \pm 8.8$ vs. $0.6 \pm 1.4 \mathrm{ng} / \mathrm{mL}, P<0.001)$. Vitamin $\mathrm{D}$ supplementation resulted in a significant reduction in serum triglycerides (vitamin D vs. placebo groups: $-37.3 \pm 75.7$ vs. $2.2 \pm 64.4 \mathrm{mg} / \mathrm{dL}, P=0.02$ ), VLDL-C levels (vitamin D vs. placebo groups: $-16.9 \pm 34.4$ vs. $1 \pm 29.2 \mathrm{mg} / \mathrm{dL}, P=0.02$ ), and hs-CRP concentration (vitamin $\mathrm{D}$ vs. placebo groups: $-850 \pm 0.321$ vs. $-106.54 \pm 5058.3 \mathrm{ng} / \mathrm{mL}, P=0.03$ ) (Table 4). When the analyses were adjusted for baseline levels of variable, age, and baseline BMI, a significant increase was observed in HDL-C concentration $(8.3 \pm 1.6$ vs. $0.52 \pm 1.6 \mathrm{mg} / \mathrm{dL}, P=0.01)$, and $P$ value of triglycerides $(P=0.01)$, VLDL-C $(P=0.01)$, and hs-CRP $(P=0.01)$ was decreased (Table 5). We did not observe a significant effect of vitamin $\mathrm{D}$ on serum omentin-1, spexin, sICAM-1, FBS, total-cholesterol, and LDL-C concentrations (Table 4). When the analyses were adjusted for baseline levels, age, and baseline BMI, an only significant decrease was observed in sICAM-1 levels $(-316.8 \pm 76.3$ vs. $-209.3 \pm 77.6 \mathrm{ng} / \mathrm{dL}$, $P=0.01)$ (Table 5). 
TABLE 2: Comparison of the anthropometric index changes within and between groups after the intervention.

\begin{tabular}{lccccccccc}
\hline & \multicolumn{3}{c}{ Placebo group $^{1}(n=25)$} & & & \multicolumn{3}{c}{ Vitamin D group $^{2}(n=30)$} \\
& Baseline & End of the trial & Change & $P^{a}$ & Baseline & End of the trial & Change & $P^{a}$ & $P^{b}$ \\
\hline Weight $(\mathrm{kg})$ & $88.05 \pm 13.32$ & $84.10 \pm 19.94$ & $-3.95 \pm 11$ & $<0.001$ & $86.33 \pm 14$ & $85.11 \pm 13.06$ & $-1.22 \pm 2.2$ & $<0.001$ & 0.58 \\
BMI $\left(\mathrm{kg} / \mathrm{m}^{2}\right)$ & $32.16 \pm 4.35$ & $31.05 \pm 4.48$ & $-1.11 \pm 0.95$ & $<0.001$ & $32.47 \pm 4.05$ & $31.06 \pm 4.06$ & $-1.41 \pm 0.97$ & $<0.001$ & 0.7 \\
Fat percent & $39.77 \pm 9.18$ & $38.80 \pm 9.52$ & $-0.96 \pm 1.81$ & 0.04 & $40.91 \pm 8$ & $39.41 \pm 8.44$ & $-1.49 \pm 1.97$ & 0.03 & 0.7 \\
Muscle percent & $26.02 \pm 5.85$ & $26.56 \pm 5.97$ & $0.53 \pm 1.30$ & 0.3 & $26.87 \pm 5.40$ & $27.40 \pm 5.56$ & $0.73 \pm 1.7$ & 0.2 & 0.35 \\
Waist circumference & $115.89 \pm 12$ & $111.72 \pm 11.9$ & $-4.17 \pm 1.61$ & $<0.001$ & $116 \pm 10.56$ & $112.3 \pm 11.06$ & $-3.66 \pm 1.19$ & $<0.001$ & 0.52 \\
(cm) & $10.36 \pm 3.20$ & $10.08 \pm 3.06$ & $-0.27 \pm 0.77$ & 0.07 & $10.27 \pm 3.37$ & $10.23 \pm 3.35$ & $-0.48 \pm 0.71$ & 0.06 & 0.65 \\
Visceral fat & & & & & & & & &
\end{tabular}

BMI: body mass index. ${ }^{1}$ Received placebo per day during 8 weeks. ${ }^{2}$ Received 2,000 IU vitamin D per day during 8 weeks. Significant difference $(P<0.05)$. Data are presented as means \pm SDs. $P^{a}$ : comparison of within-group changes (paired-sample $t$-test). $P^{b}$ : comparison of between-group changes (independent $t$ test).

TABLE 3: Dietary intakes of study participants throughout the study.

\begin{tabular}{|c|c|c|c|}
\hline & Placebo group $^{1}(n=29)$ & Vitamin D group $^{2}(n=30)$ & $P^{*}$ \\
\hline Calorie at study baseline $(\mathrm{kcal} / \mathrm{d})$ & $3302.7 \pm 889.2$ & $3373.2 \pm 1501$ & 0.8 \\
\hline Calorie at the end of the trial $(\mathrm{kcal} / \mathrm{d})$ & $2630.4 \pm 964.5$ & $2444.3 \pm 1079.6$ & 0.4 \\
\hline Carbohydrate at study baseline (gr/d) & $282.2 \pm 74.6$ & $299.9 \pm 121.7$ & 0.6 \\
\hline Carbohydrate at the end of the trial $(\mathrm{gr} / \mathrm{d})$ & $235 \pm 83.4$ & $243.8 \pm 111.5$ & 0.7 \\
\hline Protein at study baseline $(\mathrm{gr} / \mathrm{d})$ & $89.2 \pm 37$ & $84.2 \pm 38.1$ & 0.5 \\
\hline Protein at the end of the trial $(\mathrm{gr} / \mathrm{d})$ & $82 \pm 42.3$ & $69.4 \pm 37.5$ & 0.6 \\
\hline Fat at study baseline (gr/d) & $198.4 \pm 93.1$ & $211.3 \pm 127.3$ & 0.8 \\
\hline Fat at the end of the trial $(\mathrm{gr} / \mathrm{d})$ & $143.5 \pm 89.7$ & $136.3 \pm 78.2$ & 0.8 \\
\hline SAFs at study baseline $(\mathrm{gr} / \mathrm{d})$ & $36.2 \pm 15.2$ & $38 \pm 21.1$ & 0.7 \\
\hline SAFs at the end of the trial $(\mathrm{gr} / \mathrm{d})$ & $29.2 \pm 12.6$ & $28 \pm 14$ & 0.7 \\
\hline PUFA at study baseline $(\mathrm{gr} / \mathrm{d})$ & $114.1 \pm 59.7$ & $107.2 \pm 67.8$ & 0.3 \\
\hline PUFA at the end of the trial $(\mathrm{gr} / \mathrm{d})$ & $75.7 \pm 56.2$ & $70.2 \pm 55.5$ & 0.5 \\
\hline MUFA at study baseline $(\mathrm{gr} / \mathrm{d})$ & $47.7 \pm 21.7$ & $63.3 \pm 57.5$ & 0.8 \\
\hline MUFA at the end of the trial (gr/d) & $39.5 \pm 24.8$ & $39.3 \pm 28.1$ & 0.6 \\
\hline Vitamin D at study baseline $(\mu \mathrm{g} / \mathrm{d})$ & $0.44 \pm 1$ & $1.14 \pm 1.8$ & 0.5 \\
\hline Vitamin $\mathrm{D}$ at the end of the trial $(\mu \mathrm{g} / \mathrm{d})$ & $0.33 \pm 0.96$ & $1.09 \pm 2.3$ & 0.4 \\
\hline Calcium at study baseline $(\mathrm{mg} / \mathrm{d})$ & $644 \pm 411.5$ & $600 \pm 376.5$ & 0.6 \\
\hline Calcium at the end of the trial $(\mathrm{mg} / \mathrm{d})$ & $474.8 \pm 343.3$ & $523.8 \pm 332.5$ & 0.4 \\
\hline Iron at study baseline $(\mathrm{mg} / \mathrm{d})$ & $17.5 \pm 6$ & $15.37 \pm 7$ & 0.1 \\
\hline Iron at the end of the trial $(\mathrm{mg} / \mathrm{d})$ & $14.4 \pm 6.3$ & $12.15 \pm 5$ & 0.1 \\
\hline $\mathrm{Mg}$ at study baseline $(\mathrm{mg} / \mathrm{d})$ & $237.7 \pm 134.8$ & $22 \pm 128.9$ & 0.4 \\
\hline $\mathrm{Mg}$ at the end of the trial $(\mathrm{mg} / \mathrm{d})$ & $195.9 \pm 100.2$ & $166 \pm 83.9$ & 0.2 \\
\hline $\mathrm{Na}$ at study baseline $(\mathrm{mg} / \mathrm{d})$ & $1721.2 \pm 2248.9$ & $1344.9 \pm 1341.2$ & 0.8 \\
\hline $\mathrm{Na}$ at the end of the trial (mg/d) & $1265.3 \pm 2003.7$ & $921.6 \pm 740.5$ & 0.9 \\
\hline Dietary fiber at study baseline (gr/d) & $21.9 \pm 16.3$ & $17.7 \pm 10.8$ & 0.4 \\
\hline Dietary fiber at the end of the trial $(\mathrm{gr} / \mathrm{d})$ & $19.1 \pm 16.8$ & $14.4 \pm 9.4$ & 0.5 \\
\hline
\end{tabular}

SFAs: saturated fatty acids; PUFAs: polyunsaturated fatty acids; MUFAs: monounsaturated fatty acids; Na: sodium; Mg: magnesium. ${ }^{1}$ Received placebo per day during 8 weeks. ${ }^{2}$ Received 2,000 IU vitamin D per day during 8 weeks. ${ }^{*}$ Significant difference $(P<0.05)$. Data are presented as means \pm SDs. ${ }^{*}$ Obtained from independent-sample $t$-test.

\section{Discussion}

The current study indicated that vitamin D supplementation for 8 weeks in obese and overweight participants with vitamin D deficiency who had a low-calorie diet program led to a significant reduction in serum levels of hs-CRP, sICAM1, triglycerides, and VLDL-cholesterol. However, we did not find any considerable effect of vitamin D supplementation on serum omentin-1 and spexin concentrations or anthropometric indices. To the best of our knowledge, this is the first controlled trial study that assessed the effect of vitamin D supplementation on omentin-1 and spexin levels in obese and overweight participants with vitamin D deficiency undergoing a low-calorie diet.
Omentin-1 is an adipocytokine secreted from adipose tissue and may be inversely correlated with obesity and insulin resistance or impaired glucose tolerance $[15,19,20]$. Our findings show that omentin-1 and spexin levels remained unchanged in both intervention and placebo groups. Jafari et al. [19] showed that daily consumption of $2,000 \mathrm{IU}$ vitamin D-fortified yogurt for 12 weeks in postmenopausal women with type 2 diabetes improved omentin1 level. Anti-inflammatory, antiatherogenic, and antidiabetic effects can be attributed to omentin-1 [19]. In another study, a linear relationship was observed between omentin-1 level and vitamin $\mathrm{D}$ in postmenopausal women with or without osteoporosis. This association was stronger in subjects with normal vitamin D compared with insufficient levels [21]. 
TABLE 4: Means ( \pm standard deviations) of glycemic control and markers of cardiometabolic risk at baseline and after the 8-week intervention in patients with overweight and obese that received vitamin D supplement or placebo.

\begin{tabular}{|c|c|c|c|c|c|c|c|c|c|}
\hline & & lacebo group $^{1}$ & $n=29)$ & & & Vitamin D grc & $\operatorname{oup}^{2}(n=30)$ & & \\
\hline & Baseline & $\begin{array}{c}\text { End of the } \\
\text { trial }\end{array}$ & Change & $P^{a}$ & Baseline & End of the trial & Change & $P^{a}$ & $P^{b}$ \\
\hline Serum & & & & & & & & & \\
\hline $\begin{array}{l}25(\mathrm{OH}) \mathrm{D} \\
(\mathrm{ng} / \mathrm{mL})\end{array}$ & $19.3 \pm 3.6$ & $19.9 \pm 3.5$ & $0.6 \pm 1.4$ & 0.6 & $19.8 \pm 2.6$ & $36.6 \pm 9.8$ & $16.8 \pm 8.8$ & 0.02 & $<0.001$ \\
\hline $\begin{array}{l}\text { Omentin-1 } \\
(\mathrm{mg} / \mathrm{dL})\end{array}$ & $201.7 \pm 197.6$ & $216.9 \pm 179.4$ & $15.2 \pm 229.1$ & 0.6 & $255 \pm 267.1$ & $273.5 \pm 280.11$ & $18.49 \pm 280.6$ & 0.5 & 0.75 \\
\hline $\begin{array}{l}\text { Spexin } \\
(\mathrm{pg} / \mathrm{mL})\end{array}$ & $8169.4 \pm 6753.2$ & $8355.1 \pm 6109$ & $185.7 \pm 6045.7$ & 0.9 & $8533.3 \pm 6198.2$ & $8738.2 \pm 5959.3$ & $204.9 \pm 5325.8$ & 0.6 & 0.8 \\
\hline $\begin{array}{l}\text { hs-CRP } \\
\text { (ng/mL) }\end{array}$ & $4014.54 \pm 4730.89$ & $3908 \pm 5100.4$ & $-106.54 \pm 5058.3$ & 0.6 & $3606 \pm 0.522$ & $2756 \pm 0.344$ & $-850 \pm 0.321$ & 0.02 & 0.03 \\
\hline $\begin{array}{l}\text { sICAM- } \\
1(\mathrm{ng} / \mathrm{L})\end{array}$ & $835.8 \pm 687.1$ & $644.4 \pm 368.5$ & $-191.3 \pm 729.2$ & 0.4 & $837.8 \pm 578.7$ & $530.5 \pm 389.3$ & $-307.2 \pm 466.6$ & 0.01 & 0.07 \\
\hline FBS (mg/dL) & $101.75 \pm 10.84$ & $104.20 \pm 25.12$ & $2.4 \pm 19.7$ & 0.09 & $100.70 \pm 9.86$ & $96.96 \pm 10.84$ & $-3.7 \pm 10.71$ & 0.07 & 0.2 \\
\hline $\begin{array}{l}\text { Triglycerides } \\
(\mathrm{mg} / \mathrm{dL})\end{array}$ & $180.62 \pm 74.13$ & $182.82 \pm 87.76$ & $2.2 \pm 64.4$ & 0.7 & $172.36 \pm 94.33$ & $135.06 \pm 64.5$ & $-37.3 \pm 75.7$ & 0.01 & 0.02 \\
\hline $\begin{array}{l}\text { HDL-C } \\
(\mathrm{mg} / \mathrm{dL})\end{array}$ & $51.89 \pm 10.33$ & $52.31 \pm 10.51$ & $0.41 \pm 8.8$ & 0.8 & $46.80 \pm 13.46$ & $52.2 \pm 14.31$ & $5.4 \pm 10.58$ & $<0.001$ & 0.08 \\
\hline $\begin{array}{l}\text { LDL-C } \\
(\mathrm{mg} / \mathrm{dL})\end{array}$ & $137.3 \pm 44.48$ & $141.5 \pm 38.34$ & $4.24 \pm 37.38$ & 0.59 & $139.50 \pm 74.60$ & $130.20 \pm 54.48$ & $-9.3 \pm 39.24$ & 0.45 & 0.08 \\
\hline $\begin{array}{l}\text { Total } \\
\text { cholesterol } \\
(\mathrm{mg} / \mathrm{dL})\end{array}$ & $212.34 \pm 34.11$ & $206.5 \pm 37.31$ & $-5.8 \pm 41.34$ & 0.44 & $205.13 \pm 77.93$ & $190.03 \pm 73.21$ & $-15.10 \pm 24.90$ & 0.06 & 0.6 \\
\hline $\begin{array}{l}\text { VLDL-C } \\
(\mathrm{mg} / \mathrm{dL})\end{array}$ & $82.1 \pm 33.6$ & $83.1 \pm 39.8$ & $1 \pm 29.2$ & 0.7 & $78.3 \pm 42.8$ & $61.3 \pm 29.3$ & $-16.9 \pm 34.4$ & 0.01 & 0.02 \\
\hline $\mathrm{SBP}(\mathrm{mmHg})$ & $11.51 \pm 1.47$ & $11.06 \pm 1.74$ & $-0.44 \pm 1.53$ & 0.12 & $11.26 \pm 1.72$ & $11.33 \pm 1.65$ & $0.06 \pm 1.16$ & 0.75 & 0.55 \\
\hline $\begin{array}{l}\text { DBP } \\
(\mathrm{mmHg})\end{array}$ & $8.03 \pm 1.68$ & $7.84 \pm 1.32$ & $-0.18 \pm 1.12$ & 0.58 & $8.30 \pm 1.55$ & $8.22 \pm 1.44$ & $-0.8 \pm 1.25$ & 0.76 & 0.8 \\
\hline
\end{tabular}

FBS: fasting blood sugar; HDL-C: high-density lipoprotein-cholesterol; LDL-C: low-density lipoprotein-cholesterol; VLDL-C: very-low-density lipoproteincholesterol; SBP: systolic blood pressure; DBP: diastolic blood pressure. ${ }^{1}$ Received placebo per day during 8 weeks. ${ }^{2}$ Received 2,000 IU vitamin D per day during 8 weeks. ${ }^{*}$ Significant difference $(P<0.05) . P^{a}$ : comparison of within-group changes (paired-sample $t$-test). $P^{b}$ : comparison of between-group changes (independent $t$-test). Data are presented as means \pm SDs.

TABLE 5: Adjusted changes in metabolic variables in patients with overweight and obese that received either vitamin D supplement or placebo.

\begin{tabular}{lccc}
\hline & Placebo group ${ }^{1}(n=29)$ & Vitamin D group $^{2}(n=30)$ & $P^{*}$ \\
\hline Serum 25(OH)D $(\mathrm{ng} / \mathrm{mL})$ & $0.2 \pm 0.87$ & $15 \pm 0.6$ & $<.0 .11$ \\
Omentin-1 (ng/L) & $12.4 \pm 0.11$ & $184 \pm 0.14$ & 0.1 \\
Spexin $(\mathrm{pg} / \mathrm{mL})$ & $122.3 \pm 0.15$ & $-783.96 \pm 832.12$ & 0.3 \\
hs-CRP $(\mathrm{ng} / \mathrm{mL})$ & $-95.13 \pm 754.3$ & $-316.8 \pm 76.3$ & 0.01 \\
sICAM-1(ng/L) & $-209.3 \pm 77.6$ & $97.8 \pm 2.8$ & 0.01 \\
FBS $(\mathrm{mg} / \mathrm{dL})$ & $103.2 \pm 2.8$ & $38.9 \pm 11$ & 0.1 \\
Triglycerides (mg/dL) & $5.7 \pm 11.9$ & $8.3 \pm 1.6$ & 0.01 \\
HDL-cholesterol (mg/dL) & $0.52 \pm 1.6$ & $-8.4 \pm 0.4$ & 0.01 \\
LDL-cholesterol (mg/dL) & $4.9 \pm 0.4$ & $-18.5 \pm 0.5$ & 0.1 \\
Total cholesterol (mg/dL) & $-7.4 \pm 16.8$ & $15.3 \pm 0.9$ & 0.1 \\
VLDL-C (mg/dL) & $2.1 \pm 41.2$ & 0.01 \\
\hline
\end{tabular}

FBS: fasting blood sugar; HDL-C: high-density lipoprotein-cholesterol; LDL-C: low-density lipoprotein-cholesterol; and VLDL-C: very-low-density lipoprotein-cholesterol. ${ }^{1}$ Received placebo per day during 8 weeks. ${ }^{2}$ Received $2,000 \mathrm{IU}$ vitamin D per day during 8 weeks. * ${ }^{*}$ Significant difference $(P<0.05)$. ${ }^{*}$ Obtained from ANCOVA test adjusted for baseline values, age, and baseline BMI. Data are presented as means \pm SDs.

Moreno-Navarrete et al. [22] indicated that the inverse relationship between obesity and omentin-1 may be due to a downregulation of insulin gene expression. Although the desirable effects of omentin-1 have been observed in only a few studies, further studies are needed to confirm these effects and to identify more omentin-1.
In the current study, no significant differences were observed regarding the spexin levels between groups, at the end of the study. Investigations showed that spexin concentrations were found to be significantly lower in obese children [23] and adults with severe obesity [5]. Furthermore, a downregulation of spexin gene expression was 
identified in obese human fat tissue compared with nonobese individuals [24]. Decreased expression of spexin gene due to elevated serum leptin levels in obese individuals is associated with impaired intake foods and energy metabolism [5]. On the other hand, it has been suggested that spexin can act as a satiety factor [5]. Thus, adipose tissue accumulation and reduction of spexin in obesity may impair control of food intake and lack of awareness of satiety [5]. There was no significant difference between the two groups in terms of weight and fat percent; it might be an interpretation for no significant change in spexin levels after the intervention.

Vitamin D supplementation in obese and overweight adults with vitamin $\mathrm{D}$ deficiency under a low-calorie diet reduced hs-CRP concentrations significantly. Our findings are consistent with those of Asemi et al. [25] who reported that vitamin $\mathrm{D}$ supplementation $(400 \mathrm{IU} / \mathrm{d})$ resulted in a significant decrease in serum hs-CRP in 48 pregnant women aged $18-40 \mathrm{y}$ old at 25 weeks of gestation. In addition, in Sharifi et al.'s study [26], supplementation with one oral pearl consisting of 50,000 IU vitamin D3, every 14 for 120 days, had beneficial effects on hs-CRP level.

Contrary to our finding, a previous study showed that oral cholecalciferol supplementation (100,000 IU bolus followed by 4,000 IU daily) for 16 weeks among healthy overweight and obese individuals with vitamin D deficiency had no significant effects on proinflammatory markers and the nuclear factor kappa B (NF- $\kappa \mathrm{B})$ activity. They also mentioned that despite the significant increase found in serum $25(\mathrm{OH}) \mathrm{D}$ concentrations in the vitamin D group, no considerable alterations were observed in hs-CRP levels before and after the adjustment [27]. Inconsistent with our finding with respect to the significant changes in hs-CRP levels, the results of a recent meta-analysis have shown that vitamin D supplementation had no significant influence on hs-CRP levels in overweight and obese population [28]. In general, principal physiologic properties of calcitriol on cardiomyocytes, vascular smooth muscle cells, and the vascular endothelium might be related to the protective effects of vitamin D on cardiovascular disease [29]. In fact, it has been shown that 1,25-dihydroxy vitamin $\mathrm{D}$, as the active form of vitamin $\mathrm{D}$, could reduce the hs-CRP concentrations via inhibiting the NF- $\kappa \mathrm{B}$ pathway [30]. We observed that before the adjusted analysis, the supplementation of vitamin $\mathrm{D}$ had no significant effect on sICAM-1. However, a significant decrease was observed for sICAM-1 after adjusting for baseline levels, BMI, and age as the confounding variables. With regard to the relation between vitamin $\mathrm{D}$ and soluble endothelial cell adhesion molecules as the biomarkers of endothelial dysfunction, it was found that sICAM-1 might be associated with hypovitaminosis D [31]. In line with our study finding, vitamin $\mathrm{D}$ supplementation $(50,000 \mathrm{IU}$ vitamin D weekly for 12 weeks and then with the same dose every 3 weeks for 3 months) in end-stage renal disease (ESRD) patients, reduced the serum levels of ICAM and VCAM in the treatment group significantly [31]. Another study indicated that intramuscular administration 300000 IU vitamin D supplement in type 2 diabetes (T2D) patients with ischemic heart disease had no significant changes in the levels of ICAM-1 and VCAM-1 [32]. Contrary to our results, a previous parallel-group, double-blind, placebo-controlled randomized controlled trial showed that a 12-month intervention of vitamin $\mathrm{D}$ supplementation (400 IU or 1,000 IU per day) had no effect on inflammatory markers such as hs-CRP and sICAM-1 in healthy postmenopausal women [33]. Elevated levels of ICAM reflect the increased risk of CVD. In fact, the existence of cardiovascular risk factors such as insulin resistance, atherogenic dyslipidemia, hypertension, and inflammation in obese population is accompanied by the elevated levels of soluble adhesion molecules [34]. In view of the fact, normal vitamin $\mathrm{D}$ levels and a decrease in s-ICAM-1 concentration can be effective in improving endothelial function and reducing the risk of CVD [31].

In the present study, anthropometric indices including height, body weight, BMI, fat percent, and WC significantly decreased after 8-week intervention in both groups, but none of the variable indicated significant differences between vitamin D and placebo groups. Consistent with our study, Jafari-Sfidvajani et al. [35] did not find significant differences in anthropometric indices among women with polycystic ovary syndrome (PCOS) patients in two groups including $(50,000 \mathrm{IU} /$ week $)$ vitamin $\mathrm{D}_{3}+$ weight-loss diet or placebo + weight-loss diet. In addition, in line with our results, Lorvand Amiri et al. [36] reported no significant differences in anthropometric parameters among 73 nonalcoholic fatty liver disease participants with insufficient concentrations of $25(\mathrm{OH}) \mathrm{D}$ at baseline who received $25 \mu \mathrm{g}$ vitamin $\mathrm{D}_{3}$ /day or placebo for 12 weeks while participating in a weight-loss program. In agreement with our study, Salehpour et al. [37] accomplished a 12-week intervention and stated that cholecalciferol supplementation did not have any significant effect on body composition in both healthy and obese women compared to the placebo group, but a low significant decrease was observed in body fat mass. However, Wamberg et al. [38] who evaluated the long-term effects of vitamin $\mathrm{D}$ supplementation on body fat accumulation, inflammatory markers, and metabolic risk factors in obese adults with low vitamin D levels for 26 weeks did not observe any significant changes on body weight and body composition. Karonova et al. [39] reported that serum 25(OH)D level was inversely correlated with body weight, WC, and BMI in females but not in men. Furthermore, similar trends were found in the analyses of a cohort study that reported an inverse association between serum vitamin $\mathrm{D}$ and weight, BMI, and fat mass [40]. We assume that the results of the absence of significant findings regarding vitamin D supplementation and anthropometric indices could be related to the low-calorie diet program that was given to all the participants.

Findings from the current study revealed that the administration of vitamin D supplements among vitamin D-deficient obese and overweight participants led to a significant decrease in serum triglycerides and VLDL-cholesterol levels and a significant increase in serum HDL-C concentration. The correlation between hypovitaminosis $\mathrm{D}$ and the increased risk of major adverse cardiovascular disease (CVD) events has been strongly proven in 
epidemiological studies [41, 42]. In line with our study, Lorvand Amiri et al. [36] found that vitamin D supplementation in patients with nonalcoholic fatty liver disease significantly reduced triglycerides levels and increased HDLcholesterol concentrations in the intervention group. In compliance with our findings, Major et al. [43] revealed that a 15-week weight-reducing program along with a calcium plus vitamin D supplementation resulted in beneficial effects on lipoprotein profiles among overweight and obese women. The results of Nagpal et al. [44] study showed that lipid profiles of middle-aged, centrally obese, and healthy participants remained unchanged after a 6-week supplementation of cholecalciferol. Notably, the results of a previous meta-analysis indicated that vitamin D supplementation led to a significant decrease in LDL-cholesterol concentrations, while no considerable improvements were observed regarding the levels of total cholesterol, HDL-cholesterol, and triglycerides [45]. The heterogeneity between results among different studies may be related to the difference in dose and duration of vitamin D supplementation, various sample sizes, different characteristics of study populations as well as various measuring techniques and different types of formulation used in preparing the supplements, and different baseline vitamin D levels of participants.

The strength of the present study is that this is the first randomized placebo-controlled trial to investigate the effect of vitamin D supplementation on omentin-1 and spexin concentration in obese and overweight individuals with vitamin D deficiency; moreover, vitamin D levels improved in the intervention group compared to the control group. The limitations of the present study should be mentioned; the small sample size and the short duration of vitamin D intervention are the main limitations; in addition, all participants received a weight-loss intervention, so we were unable to determine the effects of vitamin $\mathrm{D}_{3}$ supplementation and low-calorie diet program separately. On the contrary, measuring the gene expression of omentin- 1 and spexin could be useful in analyzing the results, but it was not possible due to time constraints. Thus, additional randomized clinical trial studies with larger populations, longer duration on intervention, and measuring the gene expression of adipokines are required to determine the exact association of vitamin D intervention and low-calorie diet with body composition, adipokines, lipid profiles, and inflammatory markers in obese and overweight participants.

\section{Conclusion}

This study indicated a significant decrease in hs-CRP, sICAM-1, TG, and VLDL-C and a significant increase in HDL-C and serum 25(OH)D levels by vitamin D supplements in the intervention group, but no significant alterations were detected on omentin-1 and spexin concentrations; hence, further studies are needed to show vitamin $\mathrm{D}$ effects on these adipokines and confirm results.

\section{Data Availability}

The datasets used during the current study are available from the corresponding author upon reasonable request.

\section{Ethical Approval}

All processes carried out in studies involving human contributors were in line with the ethical standards of the institutional and/or national analysis committee and with the 1964 Declaration of Helsinki and its later modifications or comparable ethical standards.

\section{Consent}

All individuals expressed their consent to participate in this study.

\section{Conflicts of Interest}

The authors declare that they have no conflicts of interest.

\section{Authors' Contributions}

The research was designed by MZ and ECH. ECH and BY conducted and collected all the data. AH performed the statistical analysis. MZ, ECH, and ER wrote the manuscript. The final manuscript was read and approved by all the authors.

\section{Acknowledgments}

This study was supported by the Iran University of Medical Sciences (IUMS) (Grant no. 26191).

\section{References}

[1] P. Trayhurn, C. Bing, and I. S. Wood, "Adipose tissue and adipokines-energy regulation from the human perspective," The Journal of Nutrition, vol. 136, no. 7, pp. 1935S-1939S, 2006.

[2] J. M. Friedman, "Causes and control of excess body fat," Nature, vol. 459, no. 7245, pp. 340-342, 2009.

[3] M. S. Ellulu, I. Patimah, H. Khaza'ai, A. Rahmat, and Y. Abed, "Obesity and inflammation: the linking mechanism and the complications," Archives of Medical Science, vol. 4, no. 4, p. 851, 2017.

[4] M. A. Abbas, "Physiological functions of Vitamin D in adipose tissue," The Journal of Steroid Biochemistry and Molecular Biology, vol. 165, pp. 369-381, 2017.

[5] J. L. Walewski, F. Ge, H. Lobdell et al., "Spexin is a novel human peptide that reduces adipocyte uptake of long chain fatty acids and causes weight loss in rodents with diet-induced obesity," Obesity, vol. 22, no. 7, pp. 1643-1652, 2014.

[6] J.-Y. Zhou, L. Chan, and S.-W. Zhou, "Omentin: linking metabolic syndrome and cardiovascular disease," Current Vascular Pharmacology, vol. 12, no. 1, pp. 136-143, 2014.

[7] V. d. O. Leal and D. Mafra, "Adipokines in obesity," Clinica Chimica Acta, vol. 419, pp. 87-94, 2013.

[8] M. Dinca, M.-C. Serban, A. Sahebkar et al., "Does vitamin D supplementation alter plasma adipokines concentrations? A systematic review and meta-analysis of randomized controlled trials," Pharmacological Research, vol. 107, pp. 360371, 2016.

[9] R. C. Strange, K. E. Shipman, and S. Ramachandran, "Metabolic syndrome: a review of the role of vitamin D in mediating susceptibility and outcome," World Journal of Diabetes, vol. 6, no. 7, p. 896, 2015. 
[10] C. Duggan, J. de Dieu Tapsoba, C. Mason et al., "Effect of vitamin D3 supplementation in combination with weight loss on inflammatory biomarkers in postmenopausal women: a randomized controlled trial," Cancer Prevention Research, vol. 8, no. 7, pp. 628-635, 2015.

[11] P. Seshadri, F. F. Samaha, L. Stern, R. S. Ahima, D. Daily, and N. Iqbal, "Adipocytokine changes caused by low-carbohydrate compared to conventional diets in obesity," Metabolic Syndrome and Related Disorders, vol. 3, no. 1, pp. 66-74, 2005.

[12] W.-S. Yang, W.-J. Lee, T. Funahashi et al., "Weight reduction increases plasma levels of an adipose-derived anti-inflammatory protein, adiponectin," The Journal of Clinical Endocrinology \& Metabolism, vol. 86, no. 8, pp. 3815-3819, 2001.

[13] P. Dandona, R. Weinstock, K. Thusu, E. Abdel-Rahman, A. Aljada, and T. Wadden, "Tumor necrosis factor- in sera of obese patients: fall with weight loss," Journal of Clinical Endocrinology \& Metabolism, vol. 83, no. 8, pp. 2907-2910, 1998.

[14] P. Prasad and A. Kochhar, "Interplay of vitamin D and metabolic syndrome: a review," Diabetes \& Metabolic Syndrome: Clinical Research \& Reviews, vol. 10, no. 2, pp. 105-112, 2016.

[15] H.-Y. Pan, L. Guo, and Q. Li, "Changes of serum omentin-1 levels in normal subjects and in patients with impaired glucose regulation and with newly diagnosed and untreated type 2 diabetes," Diabetes Research and Clinical Practice, vol. 88, no. 1, pp. 29-33, 2010.

[16] P. Ziccardi, F. Nappo, G. Giugliano et al., "Reduction of inflammatory cytokine concentrations and improvement of endothelial functions in obese women after weight loss over one year," Circulation, vol. 105, no. 7, pp. 804-809, 2002.

[17] E. Hejazi, R. Amani, N. SharafodinZadeh, and B. Cheraghian, "Comparison of antioxidant status and vitamin D levels between multiple sclerosis patients and healthy matched subjects," Multiple Sclerosis International, vol. 2014, Article ID 539854, 5 pages, 2014.

[18] A. R. Webb, "Who, what, where and when-influences on cutaneous vitamin D synthesis," Progress in Biophysics and Molecular Biology, vol. 92, no. 1, pp. 17-25, 2006.

[19] T. Jafari, E. Faghihimani, A. Feizi et al., "Effects of vitamin D-fortified low fat yogurt on glycemic status, anthropometric indexes, inflammation, and bone turnover in diabetic postmenopausal women: a randomised controlled clinical trial," Clinical Nutrition, vol. 35, no. 1, pp. 67-76, 2016.

[20] R.-Z. Yang, M.-J. Lee, H. Hu et al., "Identification of omentin as a novel depot-specific adipokine in human adipose tissue: possible role in modulating insulin action," American Journal of Physiology-Endocrinology and Metabolism, vol. 290, no. 6, pp. E1253-E1261, 2006.

[21] O. Dikker, S. Bekpinar, G. Ozdemirler et al., "Evaluation of the relation between omentin-1 and vitamin D in postmenopausal women with or without osteoporosis," Experimental and Clinical Endocrinology \& Diabetes, vol. 126, no. 5, pp. 316-320, 2017.

[22] J. Moreno-Navarrete, V. Catalán, F. Ortega et al., "Circulating omentin concentration increases after weight loss," Nutrition \& Metabolism, vol. 7, no. 1, p. 27, 2010.

[23] S. Kumar, J. Hossain, N. Nader, R. Aguirre, S. Sriram, and P. B. Balagopal, "Decreased circulating levels of spexin in obese children," The Journal of Clinical Endocrinology \& Metabolism, vol. 101, no. 7, pp. 2931-2936, 2016.

[24] J. L. Walewski, F. Ge, M. Gagner et al., "Adipocyte accumulation of long-chain fatty acids in obesity is multifactorial, resulting from increased fatty acid uptake and decreased activity of genes involved in fat utilization," Obesity Surgery, vol. 20, no. 1, pp. 93-107, 2010.

[25] Z. Asemi, M. Samimi, Z. Tabassi, H. Shakeri, and A. Esmaillzadeh, "Vitamin D supplementation affects serum high-sensitivity C-reactive protein, insulin resistance, and biomarkers of oxidative stress in pregnant women," The Journal of Nutrition, vol. 143, no. 9, pp. 1432-1438, 2013.

[26] N. Sharifi, R. Amani, E. Hajiani, and B. Cheraghian, "Does vitamin $\mathrm{D}$ improve liver enzymes, oxidative stress, and inflammatory biomarkers in adults with non-alcoholic fatty liver disease? A randomized clinical trial," Endocrine, vol. 47, no. 1, pp. 70-80, 2014.

[27] A. Mousa, N. Naderpoor, and J. Johnson, "Effect of vitamin D supplementation on inflammation and nuclear factor kappa-B activity in overweight/obese adults: a randomized placebocontrolled trial," vol. 7, no. 1, p. 15154, 2017.

[28] M. Jamka, M. Woźniewicz, J. Walkowiak, P. Bogdański, J. Jeszka, and M. Stelmach-Mardas, "The effect of vitamin D supplementation on selected inflammatory biomarkers in obese and overweight subjects: a systematic review with metaanalysis," European Journal of Nutrition, vol. 55, no. 6, pp. 2163-2176, 2016.

[29] A. Zittermann, S. Frisch, H. K. Berthold et al., "Vitamin D supplementation enhances the beneficial effects of weight loss on cardiovascular disease risk markers," The American Journal of Clinical Nutrition, vol. 89, no. 5, pp. 1321-1327, 2009.

[30] S. A. Tabatabaeizadeh, A. Avan, A. Bahrami et al., "High-dose supplementation of vitamin D affects measures of systemic inflammation: reductions in High-sensitivity C-reactive Protein level and neutrophil to lymphocyte ratio (NLR) distribution," Journal of Cellular Biochemistry, vol. 118, no. 12, pp. 4317-4322, 2017.

[31] A. E. Naeini, F. Moeinzadeh, S. Vahdat, A. Ahmadi, Z. P. Hedayati, and S. Shahzeidi, "The effect of vitamin D administration on intracellular adhesion molecule-1 and vascular cell adhesion molecule-1 levels in hemodialysis patients: a placebo-controlled, double-blinded clinical trial," Journal of Research in Pharmacy Practice, vol. 6, no. 1, p. 16, 2017.

[32] A. H. Talasaz, E. Shaseb, M. Tohidi, F. Hadaegh, H. Ariannejad, and M. Abbasinazari, "Effects of parenteral vitamin $\mathrm{D}$ on the biomarkers of the endothelial function in patients with type 2 diabetes and ischemic heart disease: a randomized clinical trial," Iranian Journal of Pharmaceutical Research, vol. 17, no. 2, p. 187, 2018.

[33] A. D. Wood, K. R. Secombes, F. Thies et al., "Vitamin D3 supplementation has no effect on conventional cardiovascular risk factors: a parallel-group, double-blind, placebo-controlled rct," The Journal of Clinical Endocrinology \& Metabolism, vol. 97, no. 10, pp. 3557-3568, 2012.

[34] B. Ilincic, E. Stokic, Z. Stosic et al., "Vitamin D status and circulating biomarkers of endothelial dysfunction and inflammation in non-diabetic obese individuals: a pilot study," Archives of Medical Science, vol. 13, no. 1, pp. 53-60, 2017.

[35] S. Jafari-Sfidvajani, R. Ahangari, M. Hozoori, H. MozaffariKhosravi, H. Fallahzadeh, and A. Nadjarzadeh, "The effect of vitamin D supplementation in combination with low-calorie diet on anthropometric indices and androgen hormones in women with polycystic ovary syndrome: a double-blind, randomized, placebo-controlled trial," Journal of Endocrinological Investigation, vol. 41, no. 5, pp. 597-607, 2018.

[36] H. Lorvand Amiri, S. Agah, S. N. Mousavi, A. F. Hosseini, and F. Shidfar, "Regression of non-alcoholic fatty liver by vitamin 
D supplement: a double-blind randomized controlled clinical trial," Archives of Iranian Medicine, vol. 19, no. 9, pp. 631-638, 2016.

[37] A. Salehpour, F. Hosseinpanah, F. Shidfar et al., "A 12-week double-blind randomized clinical trial of vitamin D 3 supplementation on body fat mass in healthy overweight and obese women," Nutrition Journal, vol. 11, no. 1, p. 1, 2012.

[38] L. Wamberg, U. Kampmann, H. Stødkilde-Jørgensen, L. Rejnmark, S. B. Pedersen, and B. Richelsen, "Effects of vitamin $\mathrm{D}$ supplementation on body fat accumulation, inflammation, and metabolic risk factors in obese adults with low vitamin D levels - results from a randomized trial," European Journal of Internal Medicine, vol. 24, no. 7, pp. 644-649, 2013.

[39] T. Karonova, O. Belyaeva, E. Jude et al., "Serum $25(\mathrm{OH})$ D and adipokines levels in people with abdominal obesity," The Journal of Steroid Biochemistry and Molecular Biology, vol. 175, pp. 170-176, 2016.

[40] N. Vilarrasa, J. Maravall, A. Estepa et al., "Low 25-hydroxyvitamin D concentrations in obese women: their clinical significance and relationship with anthropometric and body composition variables," Journal of Endocrinological Investigation, vol. 30, no. 8, pp. 653-658, 2007.

[41] T. J. Wang, M. J. Pencina, S. L. Booth et al., "Vitamin D deficiency and risk of cardiovascular disease," Circulation, vol. 117 , no. 4 , pp. 503-511, 2008.

[42] J. Parker, O. Hashmi, D. Dutton et al., "Levels of vitamin D and cardiometabolic disorders: systematic review and metaanalysis," Maturitas, vol. 65, no. 3, pp. 225-236, 2010.

[43] G. C. Major, F. Alarie, J. Doré, S. Phouttama, and A. Tremblay, "Supplementation with calcium+ vitamin D enhances the beneficial effect of weight loss on plasma lipid and lipoprotein concentrations," The American Journal of Clinical Nutrition, vol. 85, no. 1, pp. 54-59, 2007.

[44] J. Nagpal, J. N. Pande, and A. Bhartia, "A double-blind, randomized, placebo-controlled trial of the short-term effect of vitamin D3supplementation on insulin sensitivity in apparently healthy, middle-aged, centrally obese men," Diabetic Medicine, vol. 26, no. 1, pp. 19-27, 2009.

[45] H. Wang, N. Xia, Y. Yang, and D.-Q. Peng, "Influence of vitamin D supplementation on plasma lipid profiles: a metaanalysis of randomized controlled trials," Lipids in Health and Disease, vol. 11, no. 1, p. 42, 2012. 